BUHEP-96-18

July 1996

\title{
Critical behavior and monopole density in U(1) lattice gauge theory *
}

\author{
Werner Kerler ${ }^{\mathrm{a}}$, Claudio Rebbi ${ }^{\mathrm{b}}$, and Andreas Weber ${ }^{\mathrm{a}}$ \\ ${ }^{a}$ Fachbereich Physik, Universität Marburg, \\ D-35032 Marburg, Germany \\ ${ }^{\mathrm{b}}$ Department of Physics, Boston University, \\ Boston, MA 02215, USA
}

Our study of the energy distribution has shown that the strength of the first order transition in the fourdimensional compact $U(1)$ lattice gauge theory decreases when the coupling $\lambda$ of the monopole term increases. The disappearance of the energy gap for sufficiently large values of $\lambda$ indicates that the transition ultimately becomes of second order. In our present investigation, based on a finite-size analysis, we show that already at $\lambda=0.9$ the critical exponent is characteristic of a second-order transition. Interestingly, this exponent turns out to be definitely different from that of the Gaussian case. We observe that the monopole density becomes constant in the second order region. In addition we find the rather surprising result that the phase transition persists up to very large values of $\lambda$, where the transition moves to (large) negative $\beta$.

\section{INTRODUCTION}

We investigate the compact $\mathrm{U}(1)$ lattice gauge theory in four dimensions with the Wilson action supplemented by a monopole term [1]

$$
S=\beta \sum_{\mu>\nu, x}\left(1-\cos \Theta_{\mu \nu, x}\right)+\lambda \sum_{\rho, x}\left|M_{\rho, x}\right|
$$

where $M_{\rho, x}=\epsilon_{\rho \sigma \mu \nu}\left(\bar{\Theta}_{\mu \nu, x+\sigma}-\bar{\Theta}_{\mu \nu, x}\right) / 4 \pi$ and the physical flux $\bar{\Theta}_{\mu \nu, x} \in[-\pi, \pi)$ is related to the plaquette angle $\Theta_{\mu \nu, x} \in(-4 \pi, 4 \pi)$ by $\Theta_{\mu \nu, x}=$ $\bar{\Theta}_{\mu \nu, x}+2 \pi n_{\mu \nu, x}$ [2]. We use periodic boundary conditions.

Our studies of the energy distribution [3, 1 , have shown that the strength of the first order transition decreases with $\lambda$ so that ultimately the transition becomes of second order. This observation has enabled us to develop an algorithm by which simulations have become possible also on big lattices [4]. In this work we study the properties of the second order phase transition in detail. Because the monopoles play a fundamental role in the dynamics of the phase transition, the addition

\footnotetext{
*Contribution to LATTICE 96, International Symposium on Lattice Field Theory, St. Louis, USA. Supported in part under DFG grants Ke 250/7-2 and 250/12-1 and under DOE grant DE-FG02-91ER40676
}

of a monopole term to the action appears particularly well suited for exploring critical properties of the system appropriate for a continuum limit.

\section{PHASE TRANSITION LINE}

In Ref. 11 Barber and Shrock observed that there is a shift of the transition point if $\lambda$ is varied. The effects of a complete suppression of monopoles have also been studied [5,6]. In our previous investigations we have determined the location of the phase transition over a wide range of values in $(\beta, \lambda)$ space. Now we wish to clarify what happens at very large $\lambda$. For this purpose we determine the critical points at values of $\lambda$ substantially larger than previously considered and allow $\beta$ to take negative values.

In order to keep the computational cost for this study within bearable limits we have used our topological characterization of the phases [3, 7]. It is based on the fact that there is an infinite network of monopole current lines in the confining phase and no such network in the Coulomb phase. On finite lattices "infinite" is to be defined in accordance with the boundary conditions [8]. For periodic boundary conditions "infinite" is equivalent to "topologically nontrivial in all direc- 
tions". Since the analysis of a single configuration (or of a few configurations, because of finite size effects, when close to the critical point) is already sufficient to identify the phase, this characterization permits to find the transition region rather quickly.

It is to be remembered that on finite lattices different order parameters lead to slightly different critical $\beta$. On an $8^{4}$ lattice the maximum of the specific heat and our topological order parameter give values 1.0075(1) and 1.0074(2) for $\lambda=0$, and $0.3870(5)$ and $0.372(3)$ for $\lambda=0.9$, respectively. To determine the location of the maximum of the specific heat for larger $\lambda$ in an efficient way we first determine the critical $\beta$ from the topological order parameter and then find the maximum of the specific heat in an easy second step.

In Figure 1 we show our results for the location of the phase transition $\beta_{C}$, defined by the maximum of the specific heat, for values of $\lambda$ ranging up to 1.3. It can be seen that the phase transition line continues to negative $\beta$.

Using the topological order parameter we could follow the line of phase transitions up to still much larger $\lambda$ : from $\lambda=1.4$ where $\beta_{C}=-0.52(2)$ to $\lambda=10$ where $\beta_{C}$ is approximately -1000 . It is to be emphasized that the characteristic topological properties of the phases have been found to be fully present throughout the range of the investigation. Thus we have the remarkable result that both phases are still present all the way up to very large $\lambda$.

We observe that finite size effects increase with $\lambda$. This is indicated by the fact that the transition region becomes broader. The width of the peak of the specific heat increases by roughly a factor of 4 from $\lambda=0$ to $\lambda=0.9$ and from $\lambda=0.9$ to $\lambda=1.3$. Similarly, from the topological order parameter, we see an increase by a factor of approximately 6 from $\lambda=0$ to $\lambda=0.9$. A further indication of larger finite size effects is that the height of the specific-heat maximum decreases with $\lambda$. The decrease is roughly by a factor of 15 from $\lambda=0$ to $\lambda=0.9$ and from $\lambda=0.9$ to $\lambda=1.3$. Thus one also sees that for very large $\lambda$ a precise determination of the location of the maximum of the specific heat becomes cumbersome.

\section{MONOPOLE NUMBER DENSITY}

At smaller $\lambda$ we have observed previously [3] that the monopole number density in the Coulomb phase is roughly constant, while in the confining phase it decreases rapidly towards this constant with increasing $\lambda$. Figure 2 gives the monopole number density along the transition line. It can be seen that starting at $\lambda=0.9$ the density becomes constant within the errors of the simulation.

From the behavior exhibited in Figure 2 we conclude that the monopole number density becomes constant, in the second order region. Indeed (as pointed out in Sect. 4) the critical behavior characteristic of a second order transition properly occurs for $\lambda=0.9$ (while for $\lambda=0.8$ there are still deviations from scaling).

\section{CRITICAL BEHAVIOR}

In order corroborate our observation that for large $\lambda$ the phase transition becomes of second order we have investigated the finite-size scaling behavior of the maximum of the specific heat $C_{\max }$. It is expected to be

$C_{\max } \sim L^{d}$

if the phase transition is of first order and

$C_{\max } \sim L^{\frac{\alpha}{\nu}}$

if it is of second order, where $\alpha$ is the critical exponent of the specific heat and $\nu$ the critical exponent of the correlation length.

In Figure 3 we present the simulation results for $C_{\max }$ which we have obtained on lattices with $L$ $=6,8,10,12$ for $\lambda=0.9$ at the corresponding values of $\beta_{C}$. The fit to these data gives

$\frac{\alpha}{\nu}=0.485(35)$

Clearly this is quite far from 4 and thus the transition not of first order. 
By the hyperscaling relation $\alpha=2-d \nu$ we find

$\nu=0.446(5)$

It is interesting to note that (4.5) is clearly different from the value $\frac{1}{2}$ of the Gaussian case.

The value in Eq. (4.5) might be compared with $\nu=0.36(1)$ obtained recently [9] in a sphere-like geometry. Further it should be remembered that values between 0.33 and 0.50 have been found, in different contexts, in works done in the eighties 10.11.

The critical $\beta$ is expected to behave as

$\beta_{C}(L)=\beta_{C}(\infty)+a L^{-\frac{1}{\nu}}$

From this relation, using the value in Eq. (4.5) and our data for $\beta_{C}(L)$ at $\lambda=0.9$, we get $\beta_{C}(\infty)$ $=0.4059(5)$ and $a=-1.99(6)$.

We have also performed simulations for $\lambda=0.8$ on lattices with $L=6,8,10$. It turns out that $C_{\max }$ does not yet scale in this case.

As a next step simulations at some value of $\lambda$ larger than 0.9 appear important in order to see whether the value of Eq. (4.5) is universal. In any case our results suggest that a "line of fixed points along which a scale-invariant continuum limit will result" [2] occurs with the action (1.1) for sufficiently large values of $\lambda$.

\section{REFERENCES}

1. J.S. Barber and R.E. Shrock, Nucl. Phys. B 257 (1985) 515.

2. T. DeGrand and D. Toussaint, Phys. Rev. D 22 (1980) 2478.

3. W. Kerler, C. Rebbi and A. Weber, Phys. Rev. D 50 (1994) 6984.

4. W. Kerler, C. Rebbi and A. Weber, Nucl. Phys. B 450 (1995) 452.

5. J.S. Barber, R.E. Shrock and R. Schrader, Phys. Lett. B152 (1985) 221.

6. V.G. Bornyakov, V.K. Mitrjushkin and M. Müller-Preussker, Nucl. Phys. B (Proc. Suppl.) 30 (1993) 587.

7. W. Kerler, C. Rebbi and A. Weber, Phys. Lett. B 348 (1995) 565.

8. W. Kerler, C. Rebbi and A. Weber, Phys. Lett. B, to be published, (1996).

9. Ch. Hoelbling, A. Jakovac, J. Jersák, C.B. Lang and T. Neuhaus, Nucl. Phys. B (Proc. Suppl.) 47 (1996) 815.

10. R. Gupta, M.A. Novotny and R. Cordery, Phys. Lett B 172 (1986) 86, and references therein.

11. C.B. Lang, Nucl. Phys. B 280 (1987) 255, and references therein.

12. G. Bhanot, Phys. Rev. D 24 (1981) 461. 


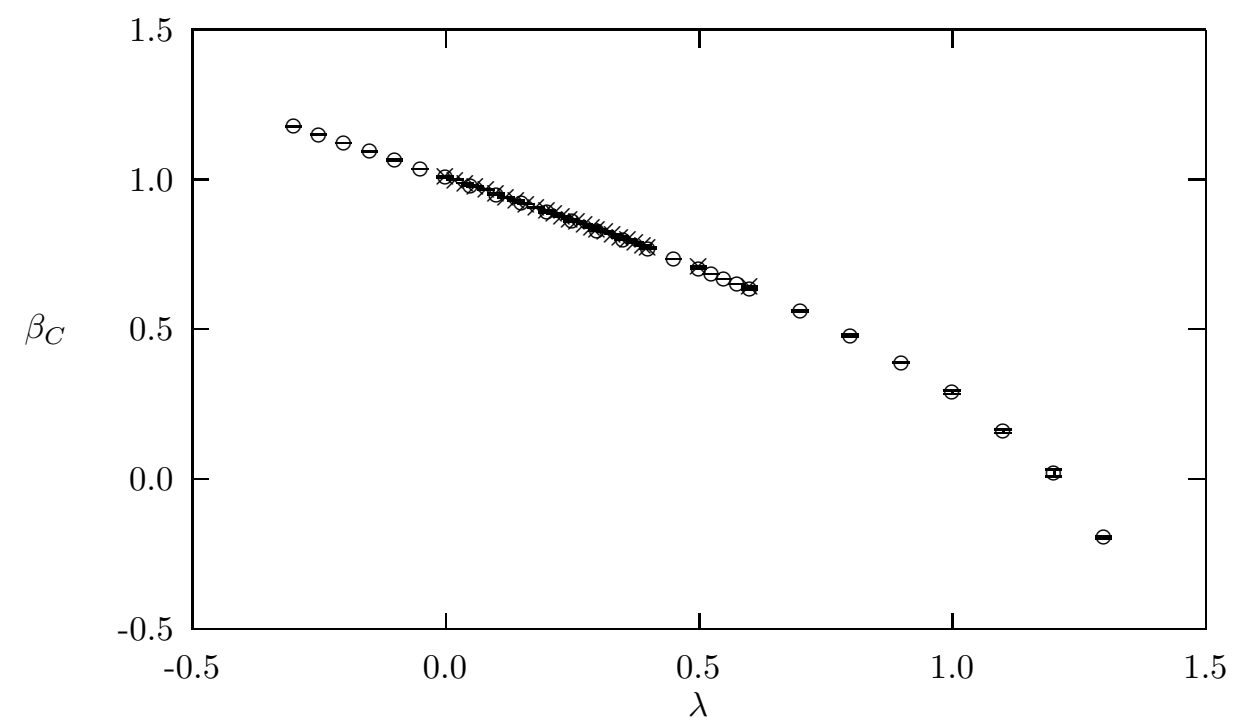

Figure 1. Location of phase transition $\beta_{C}$ as function of $\lambda$ for $8^{4}$ (circles) and $16^{4}$ (crosses) lattices.

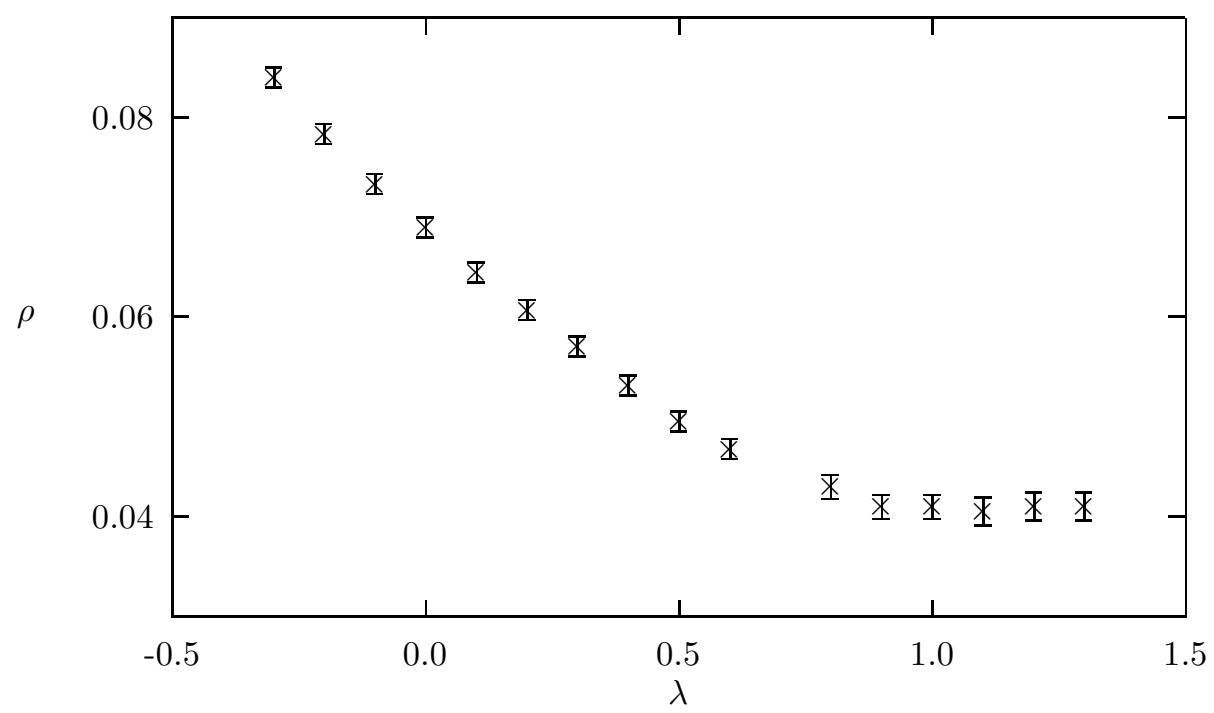

Figure 2. Monopole density as function of $\lambda$. 


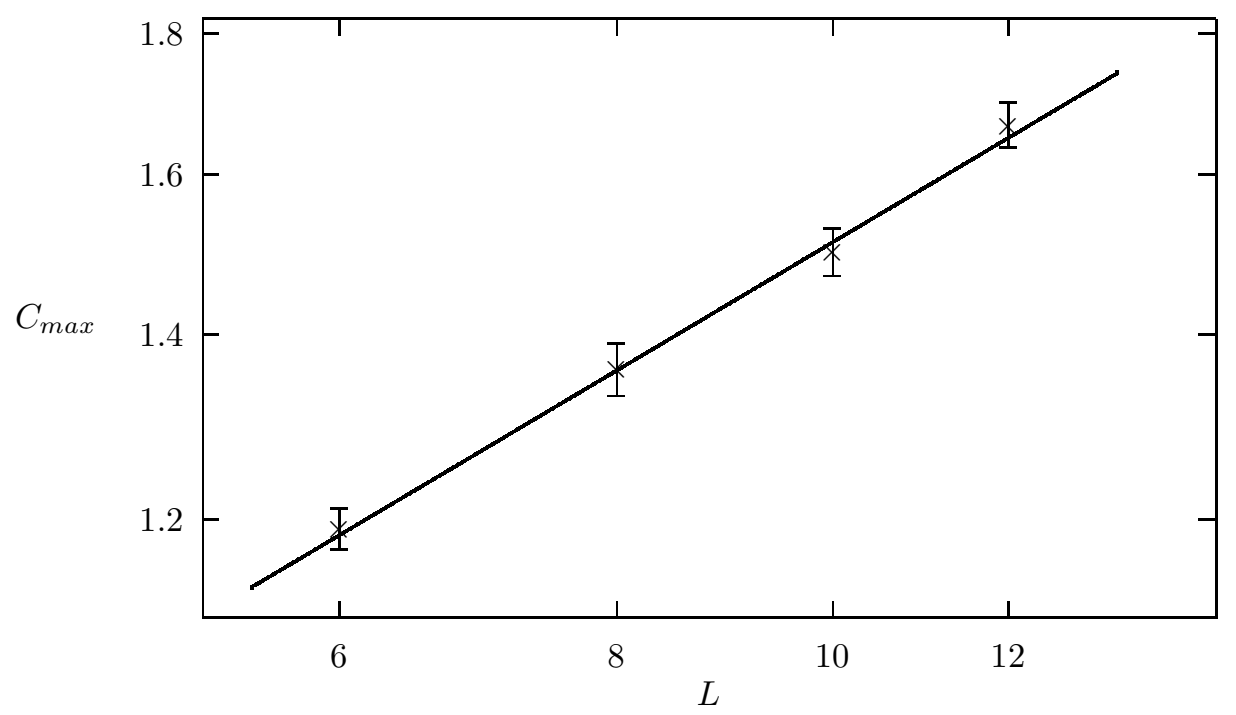

Figure 3. $C_{\max }$ versus $L$ for $\lambda=0.9$ at $\beta_{C}$. 